\title{
ON THE PROFILE OF SOLUTIONS WITH TIME-DEPENDENT SINGULARITIES FOR THE HEAT EQUATION
}

\author{
TORU KAN AND JIN TAKAHASHI
}

\begin{abstract}
Let $N \geq 2, T \in(0, \infty]$ and $\xi \in C\left(0, T ; \mathbf{R}^{N}\right)$. Under some regularity condition for $\xi$, it is known that the heat equation

$$
u_{t}-\Delta u=0, \quad x \in \mathbf{R}^{N} \backslash\{\xi(t)\}, \quad t \in(0, T)
$$

has a solution behaving like the fundamental solution of the Laplace equation as $x \rightarrow \xi(t)$ for any fixed $t$. In this paper we construct a singular solution whose behavior near $x=\xi(t)$ suddenly changes from that of the fundamental solution of the Laplace equation at some $t$.
\end{abstract}

\section{Introduction}

This paper is concerned with the following inhomogeneous linear heat equation.

$$
u_{t}-\Delta u=w(t) \delta_{\xi(t)} \quad \text { in } \mathbf{R}^{N} \times(0, T) .
$$

Here $N \geq 2, T \in(0, \infty], w$ is a weight function satisfying $w \in L^{1}(0, t)$ for each $t \in(0, T), \xi:(0, T) \rightarrow \mathbf{R}^{N}$ is a continuous curve and $\delta_{\xi(t)}$ is the Dirac distribution concentrated at $\xi(t) \in \mathbf{R}^{N}$. The main purpose of this paper is to investigate the behavior of a solution of (1.1) near $x=\xi(t)$.

Removability of singularities and existence of singular solutions in partial differential equations have been studied as interesting problems. As a simple example, let us consider the Laplace equation in $\Omega \backslash\{0\}$, where $\Omega$ is a neighborhood of 0 in $\mathbf{R}^{N}$. It is known that the singularity of a solution $u$ at 0 is removable, which means that $u$ can be extended as a solution in $\Omega$, if $u(x)=$ $o\left(|x|^{2-N}\right)$ for $N \geq 3$ and $u(x)=o(\log |x|)$ for $N=2$ as $x \rightarrow 0$. We immediately see that this condition is optimal since a fundamental solution of the Laplace equation is given by

2010 Mathematics Subject Classification. Primary 35K05, Secondary 35A20, 35B30, 35B40.

Key words and phrases. heat equation, time-dependent singularity, asymptotic expansion, profile of solution.

Received March 25, 2014; revised April 8, 2014. 


$$
\Psi(x):= \begin{cases}\frac{1}{N(N-2) \omega_{N}}|x|^{2-N}=\frac{\Gamma(N / 2-1)}{4 \pi^{N / 2}}|x|^{2-N} & \text { if } N \geq 3, \\ \frac{1}{2 \pi} \log \frac{1}{|x|} & \text { if } N=2,\end{cases}
$$

where $\omega_{N}$ is the volume of the unit ball in $\mathbf{R}^{N}$ and $\Gamma$ denotes the gamma function. For nonlinear elliptic equations, this kind of problems were examined by many authors, see, e.g., $[1,12,8,2,13]$ and references therein.

In the recent works by $[5,6,4]$, a condition for removability of singularities was considered in a certain class of nonlinear parabolic equations including the Fujita equation

$$
u_{t}=\Delta u+|u|^{p-1} u
$$

with $N \geq 3$ and $0 \leq p<N /(N-2)$. More precisely, it was shown that a solution $u$ of $(1.2)$ in $(\Omega \backslash\{0\}) \times(0, T)$ can be extended as a solution in $\Omega \times(0, T)$ if $u$ satisfies $u(x, t)=o\left(|x|^{2-N}\right)$ locally uniformly for $t \in(0, T)$ as $x \rightarrow 0$.

An interesting problem on singular solutions of parabolic equations is the existence of solutions with a time-dependent singularity. Here, by time-dependent singularity, we mean a singularity with respect to the space variable whose position depends on the time variable. The existence of such solutions were revealed in $[9,10]$ for the equation (1.2) with $N \geq 3$ and $p$ in a certain range. Recently, solutions with a time-dependent singularity were constructed also in the NavierStokes equation [7].

In this paper, we focus on the linear heat equation

$$
u_{t}-\Delta u=0, \quad x \in \mathbf{R}^{N} \backslash\{\xi(t)\}, \quad t \in(0, T) .
$$

For this equation, the following were shown in [11, 7]. A condition for the removability of singularities is given by $u(x, t)=o(\Psi(x-\xi(t)))$ locally uniformly for $t \in(0, T)$ as $x \rightarrow \xi(t)$, if $\xi$ is $1 / 2$-Hölder continuous. At the same time, under the condition that $\xi$ has $\alpha$-Hölder continuity with $\alpha>1 / 2$, there is a solution $\tilde{u}$ satisfying

$$
\tilde{u}(x, t)=(1+o(1)) \Psi(x-\xi(t)) \text { for every } t \in(0, T) \text { as } x \rightarrow \xi(t) .
$$

These results are analogous to the case of the Laplace equation. Our interest here is a solution which has a singularity at $x=\xi(t)$ but does not always behave like $\Psi(x-\xi(t))$ as $x \rightarrow \xi(t)$. In order to construct such a solution, we consider the equation (1.1). Then a desired solution can be found by considering two effects. One is due to the quick movement of $\xi$. We will observe that the profile of a solution of (1.1) is distorted when $\xi$ loses $\alpha$-Hölder continuity with $\alpha>1 / 2$. The other one is the weight $w$. We will show that if $w$ is forced to vanish or diverge as $t \uparrow t_{0}$, then the strength of a singularity suddenly changes at $t_{0}$.

This paper is organized as follows. In the next section, we set up our problem precisely and state main results. Section 3 is devoted to proving the 
results. In Section 4, we discuss the precise behavior at $x=\xi(t)$ in the case where $w(t) \equiv 1$ and $\xi$ is $\alpha$-Hölder continuous with $\alpha>1 / 2$.

\section{Main results}

We introduce some definitions and notation before stating our results. Let $F$ be defined by

$$
F(x, t):=\int_{0}^{t} w(s) \Phi(x-\xi(s), t-s) d s,
$$

where $\Phi$ is the heat kernel, that is, $\Phi(x, t)=(4 \pi t)^{-N / 2} e^{-|x|^{2} / 4 t}$. Note that $F$ can be defined for $x \in \mathbf{R}^{N} \backslash\{\xi(t)\}$ and $t \in(0, T)$, since

$$
\sup _{s \in(0, t)} \Phi(x-\xi(s), t-s)<+\infty
$$

provided $x \neq \xi(t)$. Moreover,

$$
\|F(\cdot, t)\|_{L^{1}\left(\mathbf{R}^{N}\right)} \leq \int_{0}^{t}|w(s)|\left(\int_{\mathbf{R}^{N}} \Phi(x-\xi(s), t-s) d x\right) d s=\int_{0}^{t}|w(s)| d s,
$$

and so $F \in L^{\infty}\left(0, t ; L^{1}\left(\mathbf{R}^{N}\right)\right)$ for any $t \in(0, T)$. We will prove that $F$ satisfies (1.1) in the distributional sense, that is, the equality

$$
\int_{0}^{T} \int_{\mathbf{R}^{N}} F(x, t)\left(-\varphi_{t}(x, t)-\Delta \varphi(x, t)\right) d x d t=\int_{0}^{T} w(t) \varphi(\xi(t), t) d t
$$

holds for all $\varphi \in C_{0}^{\infty}\left(\mathbf{R}^{N} \times(0, T)\right)$ (see Proposition 3.1 in Section 3). In particular, by the Weyl lemma for the heat equation (see, e.g., [3, Section 6]), we see that $F$ satisfies (1.3) in the classical sense. For the same reason, we also find that if $u \in L_{\text {loc }}^{1}\left(\mathbf{R}^{N} \times(0, T)\right)$ satisfies (1.1) in the distributional sense, then $u-F$ is smooth in $\mathbf{R}^{N} \times(0, T)$. This implies that the singularity of any solution of (1.1) is determined by $F$, and therefore our focus is on the behavior of $F$ as $x \rightarrow \xi(t)$.

Let $t_{0} \in(0, T)$ be fixed. As instantaneous quickness of $\xi(t)$ and weight of $w(t)$ at $t=t_{0}$, we define

$$
v_{\alpha}:=\lim _{s \uparrow t_{0}} \frac{\xi\left(t_{0}\right)-\xi(s)}{\left(t_{0}-s\right)^{\alpha}} \quad(\alpha>0), \quad w_{\beta}:=\lim _{s \uparrow t_{0}} \frac{w(s)}{\left(t_{0}-s\right)^{\beta}} \quad(\beta>-1)
$$

if their respective limits exist. Throughout this paper, we suppose that

$$
v_{\alpha} \text { and } w_{\beta} \text { exist for some } \alpha>0 \text { and } \beta>-1 \text {. }
$$

The goal of this study is to describe how the magnitude and the direction of $v_{\alpha}$ and the weight $w_{\beta}$ influence the singularity of $F\left(x, t_{0}\right)$.

Set $p:=N / 2-\beta$. If $w_{\beta}$ exists for some $\beta$ with $p<1$, then we have

$$
\left|w(s) \Phi\left(x-\xi(s), t_{0}-s\right)\right| \leq(4 \pi)^{-N / 2}|w(s)|\left(t_{0}-s\right)^{-N / 2} \leq C\left(t_{0}-s\right)^{-p}
$$


for all $x \in \mathbf{R}^{N}$ and $s \in\left(0, t_{0}\right)$. Here $C>0$ is a constant. This shows that the value of $F\left(x, t_{0}\right)$ at $x=\xi\left(t_{0}\right)$ can be defined as a finite value. Furthermore, Lebesgue's dominated convergence theorem yields

$$
\lim _{x \rightarrow \xi\left(t_{0}\right)} F\left(x, t_{0}\right)=F\left(\xi\left(t_{0}\right), t_{0}\right) .
$$

Therefore, in what follows, we only consider the case $p \geq 1$.

We perform the change of variables $z=x-\xi\left(t_{0}\right)$ and $\tau=t_{0}-s$. Then

$$
F\left(x, t_{0}\right)=(4 \pi)^{-N / 2} \int_{0}^{t_{0}} w\left(t_{0}-\tau\right) \tau^{-N / 2} \exp \left(-\frac{1}{4 \tau}\left|z+\tau^{\alpha} \gamma_{\alpha}(\tau)\right|^{2}\right) d \tau,
$$

where we write $\xi\left(t_{0}\right)-\xi\left(t_{0}-\tau\right)=\tau^{\alpha} \gamma_{\alpha}(\tau)$. Put $\rho_{\alpha}:=\left|v_{\alpha}\right|$ and $v_{\alpha}:=v_{\alpha} /\left|v_{\alpha}\right|$. We write $r=|z|, \omega=z /|z|$ and denote $\theta \in[0, \pi]$ by the angle between $\omega$ and $-v_{\alpha}$, that is, $\cos \theta=-\omega \cdot v_{\alpha}$. With this notation, we have the decomposition $\omega=$ $-(\cos \theta) v_{\alpha}+(\sin \theta) n$ for some $n \in \mathbf{R}^{N}$ with $|n|=1$ and $n \cdot v_{\alpha}=0$.

The effect of $v_{\alpha}$ is considerably different depending on $\alpha$. When $\alpha=1 / 2$, the effect of $v_{\alpha}$ appears in the coefficient of the leading term and $F\left(x, t_{0}\right)$ can lose asymptotic radial symmetry as $x \rightarrow \xi\left(t_{0}\right)$.

THEOREM 2.1. Let $\alpha=1 / 2$. Then the following (i) and (ii) hold as $z=$ $x-\xi\left(t_{0}\right) \rightarrow 0$.

(i) If $p=1$,

$$
F\left(x, t_{0}\right)=2^{1-N} \pi^{-N / 2} w_{\beta} e^{-\rho_{\alpha}^{2} / 4} \log \frac{1}{r}+o\left(\log \frac{1}{r}\right) .
$$

(ii) If $p>1$,

$$
F\left(x, t_{0}\right)=(4 \pi)^{-N / 2} w_{\beta}\left(\int_{0}^{\infty} \sigma^{p-2} e^{-(1 / 4)\left|\sqrt{\sigma} \omega+v_{\alpha}\right|^{2}} d \sigma\right) r^{-2(p-1)}+o\left(r^{-2(p-1)}\right) .
$$

Remark 2.1. Suppose that $w_{\beta}=1$ and $v_{1 / 2}=0$. Then Theorem 2.1 implies that, as $x \rightarrow \xi\left(t_{0}\right)$,

$$
\begin{aligned}
F\left(x, t_{0}\right) & = \begin{cases}(2 \pi)^{-1} \log \frac{1}{r}+o\left(\log \frac{1}{r}\right) & (N=2) \\
(4 \pi)^{-N / 2}\left(\int_{0}^{\infty} \sigma^{N / 2-2} e^{-(1 / 4) \sigma} d \sigma\right) r^{2-N}+o\left(r^{2-N}\right) & (N \geq 3)\end{cases} \\
& =(1+o(1)) \Psi(z) .
\end{aligned}
$$

Next, let us consider the case $\alpha<1 / 2$. We remark that if $v_{\alpha} \neq 0$ with some $\alpha<1 / 2$, the integral

$$
\int_{0}^{t_{0}}|w(s)|\left(t_{0}-s\right)^{-N / 2} e^{-\left|\xi\left(t_{0}\right)-\xi(s)\right|^{2} / 4\left(t_{0}-s\right)} d s=\int_{0}^{t_{0}}\left|w\left(t_{0}-\tau\right)\right| \tau^{-N / 2} e^{-(1 / 4) \tau^{-(1-2 \alpha)}\left|\gamma_{\alpha}(\tau)\right|^{2}} d \tau
$$


is finite, because the integrand is bounded by $C\left|w\left(t_{0}-\tau\right)\right|$ for some constant $C>0$. Therefore the value of $F\left(x, t_{0}\right)$ at $x=\xi\left(t_{0}\right)$ can be defined as a finite value. This fact suggests that there is some region $\mathscr{N}$ containing the point $\xi\left(t_{0}\right)$ such that $F\left(\cdot, t_{0}\right)$ is bounded in $\mathscr{N}$. The problems in this case are to find such a region $\mathscr{N}$ and to determine the asymptotic behavior of $F\left(x, t_{0}\right)$ as $x \notin \mathscr{N}$, $x \rightarrow \xi\left(t_{0}\right)$. In order to state our result, we define, for $\varepsilon>0$ and $M>0$,

$$
\begin{gathered}
S_{\varepsilon}:=\left\{z \in \mathbf{R}^{N} \backslash\{0\} ; 1-\cos \theta \geq 2 \rho_{\alpha}^{-1 / \alpha}\left(\frac{2 p-3}{2 \alpha}+1+\varepsilon\right) r^{1 / \alpha-2} \log \frac{1}{r}\right\}, \\
T_{M}:=\left\{z \in \mathbf{R}^{N} \backslash\{0\} ; 1-\cos \theta \leq M r^{1 / \alpha-2}\right\} .
\end{gathered}
$$

THEOREM 2.2. Let $\alpha \in(0,1 / 2)$ and $v_{\alpha} \neq 0$. Then the following (i) and (ii) hold.

(i) Suppose that $1 \leq p<3 / 2-\alpha$ and

$$
\xi\left(t_{0}\right)-\xi(s)=\left(t_{0}-s\right)^{\alpha} v_{\alpha}+o\left(\left(t_{0}-s\right)^{\alpha+p-1}\right)
$$

as $s \uparrow t_{0} . \quad$ Then, as $z=x-\xi\left(t_{0}\right) \rightarrow 0$,

$$
F\left(x, t_{0}\right)=F\left(\xi\left(t_{0}\right), t_{0}\right)+o(1) .
$$

(ii) Suppose that $p \geq 3 / 2-\alpha$ and

$$
\xi\left(t_{0}\right)-\xi(s)=\left(t_{0}-s\right)^{\alpha} v_{\alpha}+\left(t_{0}-s\right)^{1 / 2} \hat{v}+o\left(\left(t_{0}-s\right)^{1 / 2}\right)
$$

for some $\hat{v} \in \mathbf{R}^{N}$ as $s \uparrow t_{0}$. Then, for any $\varepsilon>0$ and $M>0$,

$$
\begin{aligned}
& F\left(x, t_{0}\right)=F\left(\xi\left(t_{0}\right), t_{0}\right)+o(1) \\
& \text { as } z=x-\xi\left(t_{0}\right) \in S_{\varepsilon}, z \rightarrow 0 \text {, and }
\end{aligned}
$$

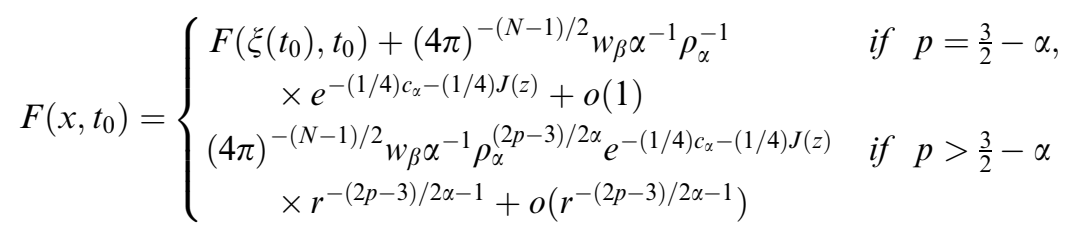

$$
\begin{aligned}
& \text { as } z=x-\xi\left(t_{0}\right) \in T_{M}, \quad z \rightarrow 0 . \quad \text { Here } J(z):=2 \rho_{\alpha}^{1 / \alpha} r^{-(1 / \alpha-2)}(1-\cos \theta)+ \\
& 2 \rho_{\alpha}^{1 / 2 \alpha}(n \cdot \hat{v}) r^{-(1 / 2 \alpha-1)} \sin \theta \text { and } c_{\alpha}:=|\hat{v}|^{2}-\left(v_{\alpha} \cdot \hat{v}\right)^{2} \text {. Furthermore, } \\
& \liminf _{x \rightarrow \xi\left(t_{0}\right)} F\left(x, t_{0}\right)=F\left(\xi\left(t_{0}\right), t_{0}\right), \\
& \limsup _{x \rightarrow \xi\left(t_{0}\right)}\left(r^{(2 p-3) / 2 \alpha+1} F\left(x, t_{0}\right)\right) \\
& = \begin{cases}F\left(\xi\left(t_{0}\right), t_{0}\right)+(4 \pi)^{-(N-1) / 2} w_{\beta} \alpha^{-1} \rho_{\alpha}^{-1} & \text { if } p=\frac{3}{2}-\alpha, \\
(4 \pi)^{-(N-1) / 2} w_{\beta} \alpha^{-1} \rho_{\alpha}^{(2 p-3) / 2 \alpha} & \text { if } p>\frac{3}{2}-\alpha .\end{cases}
\end{aligned}
$$


Remark 2.2. We can also discuss a solution which has a singularity for all $t \in \mathbf{R}$. Let $W \in L_{\text {loc }}^{1}(\mathbf{R})$ satisfy $(-t)^{-N / 2} W(t) \in L^{1}(-\infty,-1)$ and let $\Xi$ be a continuous curve in $\mathbf{R}$. We set

$$
G(x, t):=\int_{-\infty}^{t} W(s) \Phi(x-\Xi(s), t-s) d s .
$$

Then it can be shown that $G$ satisfies $G_{t}-\Delta G=W(t) \delta_{\Xi(t)}$ in $\mathbf{R}^{N} \times(-\infty, \infty)$ and the same assertions as in Theorems 2.1 and 2.2 hold by replacing $F$ with $G$. We note that in the special case $N \geq 3, W \equiv 1$ and $\Xi \equiv 0, G$ coincides $\Psi$.

\section{Proofs of theorems}

First of all, we show that the function $F$ defined by (2.1) is a solution of (1.1). Although this fact is essentially proved in [11, Section 4], we give a proof for the completion.

Proposition 3.1. F satisfies (1.1) in the distributional sense.

Proof. We fix $\varphi \in C_{0}^{\infty}\left(\mathbf{R}^{N} \times(0, T)\right)$ and take $0<\underline{t}<\bar{t}<T$ such that the support of $\varphi$ is contained in $\mathbf{R}^{N} \times(\underline{t}, \bar{t})$. It is enough to verify (2.2) under the assumption that $w$ is smooth. Indeed, choosing $\left\{w_{n}\right\}_{n=1}^{\infty} \subset C_{0}^{\infty}(\mathbf{R})$ such that $w_{n} \rightarrow w$ in $L^{1}(0, \bar{t})$ as $n \rightarrow \infty$ and putting $\tilde{F}_{n}(x, t):=\int_{0}^{t} w_{n}(s) \Phi(x-\xi(s), t-s) d s$, we have

$$
\begin{aligned}
\sup _{t \in(\underline{t}, \bar{t})}\left\|F(\cdot, t)-\tilde{F}_{n}(\cdot, t)\right\|_{L^{1}\left(\mathbf{R}^{N}\right)} & \leq \sup _{t \in(t, \bar{t})} \int_{0}^{t}\left|w(s)-w_{n}(s)\right|\|\Phi(\cdot-\xi(s), t-s)\|_{L^{1}\left(\mathbf{R}^{N}\right)} d s \\
& \leq\left\|w-w_{n}\right\|_{L^{1}(0, \bar{t})} \rightarrow 0 \quad(n \rightarrow \infty) .
\end{aligned}
$$

Therefore once we show that $\tilde{F}_{n}$ satisfies (2.2), the proof is finished by letting $n \rightarrow \infty$.

From now on we assume $w \in C_{0}^{\infty}(\mathbf{R})$. Let $h>0$ and define

$$
F_{h}(x, t):=\int_{0}^{t-h} w(s) \Phi(x-\xi(s), t-s) d s .
$$

Then we easily see that $F_{h}$ satisfies $\left(F_{h}\right)_{t}-\Delta F_{h}=w(t-h) \Phi(x-\xi(t-h), h)$ in the classical sense in $\mathbf{R}^{N} \times(h, T)$. Hence integration by parts yields

$$
\int_{0}^{T} \int_{\mathbf{R}^{N}} F_{h}\left(-\varphi_{t}-\Delta \varphi\right) d x d t=\int_{0}^{T} w(t-h)\left(\int_{\mathbf{R}^{N}} \varphi(x, t) \Phi(x-\xi(t-h), h) d x\right) d t
$$

provided $h<t . t$. Let us take the limit as $h \downarrow 0$. Then the left-hand side convergences to $\int_{0}^{T} \int_{\mathbf{R}^{N}} F\left(-\varphi_{t}-\Delta \varphi\right) d x d t$, because

$$
\begin{aligned}
\sup _{t \in(\underline{t}, \bar{t})}\left\|F(\cdot, t)-F_{h}(\cdot, t)\right\|_{L^{1}\left(\mathbf{R}^{N}\right)} & \leq \sup _{t \in(\underline{t}, \bar{t})} \int_{t-h}^{t}|w(s)|\|\Phi(\cdot-\xi(s), t-s)\|_{L^{1}\left(\mathbf{R}^{N}\right)} d s \\
& \leq\|w\|_{L^{\infty}(\mathbf{R})} h \rightarrow 0 \quad(h \downarrow 0) .
\end{aligned}
$$


It is straightforward to show that $w(t-h) \rightarrow w(t)$ and $\int_{\mathbf{R}^{N}} \varphi(x, t) \Phi(x-$ $\xi(t-h), h) d x \rightarrow \varphi(\xi(t), t)$ locally uniformly for $t \in(0, T)$ as $h \downarrow 0$. Hence we see that the right-hand side tends to $\int_{0}^{T} w(t) \varphi(\xi(t), t) d t$. Thus the proof is complete.

3.1. The case $\alpha=1 / 2$. Let us consider the case $\alpha=1 / 2$ and prove Theorem 2.1.

Proof of Theorem 2.1. We fix $\delta \in(0,1)$ and take $\tau_{0}>0$ such that $\left(w_{\beta}-\delta\right) \tau^{\beta} \leq w\left(t_{0}-\tau\right) \leq\left(w_{\beta}+\delta\right) \tau^{\beta}$ and $\rho_{\alpha}^{2}-\delta \leq\left|\gamma_{\alpha}(\tau)\right|^{2} \leq \rho_{\alpha}^{2}+\delta$ for $\tau \in\left(0, \tau_{0}\right]$. Then

$$
\left(w_{\beta}-\delta\right) I(z) \leq(4 \pi)^{N / 2} F\left(x, t_{0}\right) \leq C+\left(w_{\beta}+\delta\right) I(z),
$$

where $C>0$ is a constant and

$$
I(z):=\int_{0}^{\tau_{0}} \tau^{-p} e^{-(1 / 4 \tau)\left|z+\tau^{\alpha} \gamma_{\alpha}(\tau)\right|^{2}} d \tau .
$$

First, we assume $p=1$ and prove (i). Since

$$
r^{2}-2\left(\rho_{\alpha}^{2}+1\right) r \tau^{1 / 2}+\left(\rho_{\alpha}^{2}-\delta\right) \tau \leq\left|z+\tau^{\alpha} \gamma_{\alpha}(\tau)\right|^{2} \leq r^{2}+2\left(\rho_{\alpha}^{2}+1\right) r \tau^{1 / 2}+\left(\rho_{\alpha}^{2}+\delta\right) \tau
$$

for $\tau \in\left(0, \tau_{0}\right]$, we have

$$
\begin{aligned}
& e^{-\left(\rho_{\alpha}^{2}+\delta\right) / 4} \int_{0}^{\tau_{0}} \tau^{-1} e^{-r^{2} / 4 \tau-\left(\rho_{\alpha}^{2}+1\right) r / 2 \sqrt{\tau}} d \tau \\
& \quad \leq I(z) \leq e^{-\left(\rho_{\alpha}^{2}-\delta\right) / 4} \int_{0}^{\tau_{0}} \tau^{-1} e^{-r^{2} / 4 \tau+\left(\rho_{\alpha}^{2}+1\right) r / 2 \sqrt{\tau}} d \tau .
\end{aligned}
$$

By the change of variables $\sigma=r^{2} / \tau$ and integration by parts, we deduce that

$$
\begin{aligned}
\int_{0}^{\tau_{0}} \tau^{-1} & e^{-r^{2} / 4 \tau \pm\left(\rho_{\alpha}^{2}+1\right) r / 2 \sqrt{\tau}} d \tau \\
= & \int_{r^{2} / \tau_{0}}^{\infty} \sigma^{-1} e^{-(1 / 4) \sigma \pm(1 / 2)\left(\rho_{\alpha}^{2}+1\right) \sqrt{\sigma}} d \sigma \\
= & \left(\log \frac{\tau_{0}}{r^{2}}\right) \exp \left(-\frac{r^{2}}{4 \tau_{0}} \pm \frac{\left(\rho_{\alpha}^{2}+1\right) r}{2 \sqrt{\tau_{0}}}\right) \\
& -\int_{r^{2} / \tau_{0}}^{\infty}\left(-\frac{1}{4} \pm \frac{\rho_{\alpha}^{2}+1}{4 \sqrt{\sigma}}\right) e^{-(1 / 4) \sigma \pm(1 / 2)\left(\rho_{\alpha}^{2}+1\right) \sqrt{\sigma}} \log \sigma d \sigma \\
= & 2 \log \frac{1}{r}+O(1) \quad(z \rightarrow 0) .
\end{aligned}
$$

Thus, combining (3.1)-(3.3), we obtain (i). 

yields

Next, we show (ii). In the case $p>1$, the change of variables $\sigma=r^{2} / \tau$

$$
\left|r^{2(p-1)} I(z)\right| \leq \int_{r^{2} / \tau_{0}}^{\infty} \sigma^{p-2} e^{-(1 / 4)\left|\sqrt{\sigma} \omega+\gamma_{\alpha}\left(r^{2} / \sigma\right)\right|^{2}} d \sigma \leq C
$$

for all $r>0$ with some constant $C>0$, and

$$
\begin{aligned}
r^{2(p-1)} & I(z)-\int_{0}^{\infty} \sigma^{p-2} e^{-(1 / 4)\left|\sqrt{\sigma} \omega+v_{\alpha}\right|^{2}} d \sigma \\
\quad= & \int_{0}^{\infty} \sigma^{p-2}\left(e^{-(1 / 4)\left|\sqrt{\sigma} \omega+\gamma_{\alpha}\left(r^{2} / \sigma\right)\right|^{2}} \chi_{\left[r^{2} / \tau_{0}, \infty\right)}(\sigma)-e^{-(1 / 4)\left|\sqrt{\sigma} \omega+v_{\alpha}\right|^{2}}\right) d \sigma
\end{aligned}
$$

where $\chi_{A}$ denotes the indicator function of a set $A$. The integrand of the right-hand side convergences to 0 for each $\sigma \in(0, \infty)$ and is bounded by $C \sigma^{p-2} e^{-(1 / 8) \sigma}$. Lebesgue's dominated convergence theorem, (3.1) and (3.4) show

$$
\limsup _{z \rightarrow 0}\left|\frac{(4 \pi)^{N / 2} F\left(x, t_{0}\right)}{r^{-2(p-1)}}-w_{\beta} \int_{0}^{\infty} \sigma^{p-2} e^{-(1 / 4)\left|\sqrt{\sigma} \omega+v_{\alpha}\right|^{2}} d \sigma\right| \leq C \delta .
$$

This gives (ii).

3.2. The case $\alpha<1 / 2$. Under the assumption that $\alpha \in(0,1 / 2)$ and $v_{\alpha} \neq 0$ $\left(\rho_{\alpha}>0\right)$, we separate $F\left(x, t_{0}\right)$ into two parts as follows.

$$
\begin{aligned}
(4 \pi)^{N / 2} F\left(x, t_{0}\right) & =\int_{\left(0, \tau_{+}\right) \cup\left(\tau_{-}, t_{0}\right)}+\int_{\tau_{+}}^{\tau_{-}} w\left(t_{0}-\tau\right) \tau^{-N / 2} e^{-(1 / 4 \tau)\left|z+\tau^{\alpha} \gamma_{\alpha}(\tau)\right|^{2}} d \tau \\
& =: I_{1}(z)+I_{2}(z),
\end{aligned}
$$

where $\tau_{ \pm}:=\left\{r(1 \pm \delta)^{-1} \rho_{\alpha}^{-1}\right\}^{1 / \alpha}$ and $\delta \in(0,1)$.

To prove Theorem 2.2, we prepare some lemmas. The first one gives the behavior of $I_{1}$.

Lemma 3.1. For any fixed $\delta \in(0,1)$,

$$
\lim _{z \rightarrow 0} I_{1}(z)=(4 \pi)^{N / 2} F\left(\xi\left(t_{0}\right), t_{0}\right) .
$$

Proof. Note that $\tau_{+} \leq \tau \leq \tau_{-}$is equivalent to $\rho_{\alpha}(1-\delta) \tau^{\alpha} \leq r \leq \rho_{\alpha}(1+\delta) \tau^{\alpha}$. Hence, taking $\tau_{0}>0$ so that $\rho_{\alpha}(1-\delta / 2) \leq\left|\gamma_{\alpha}(\tau)\right| \leq \rho_{\alpha}(1+\delta / 2)$ for $\tau \in\left(0, \tau_{0}\right]$, we have

$$
\left|z+\tau^{\alpha} \gamma_{\alpha}(\tau)\right|^{2} \geq\left(r-\tau^{\alpha}\left|\gamma_{\alpha}(\tau)\right|\right)^{2} \geq \frac{\rho_{\alpha}^{2} \delta^{2}}{4} \tau^{2 \alpha}
$$


provided that $\tau \in\left(0, \tau_{+}\right] \cup\left[\tau_{-}, \tau_{0}\right]$. This implies that for all $z \in \mathbf{R}^{N}$ and $\tau \in\left(0, t_{0}\right)$,

$$
\begin{aligned}
\left|w\left(t_{0}-\tau\right)\right| \tau^{-N / 2} e^{-(1 / 4 \tau)\left|z+\tau^{\alpha} \gamma_{\alpha}(\tau)\right|^{2}} \chi_{\left(0, \tau_{+}\right) \cup\left(\tau_{-}, t_{0}\right)}(\tau) & \leq C\left|w\left(t_{0}-\tau\right)\right| \tau^{-N / 2} e^{-\left(\rho_{\alpha}^{2} \delta^{2} / 16\right) \tau^{-(1-2 \alpha)}} \\
& \leq C\left|w\left(t_{0}-\tau\right)\right|
\end{aligned}
$$

Here $C>0$ is a constant independent of $z$ and $\tau$. Since $w\left(t_{0}-\cdot\right) \in L^{1}\left(0, t_{0}\right)$, we obtain (3.5) by Lebesgue's dominated convergence theorem.

Next we consider $I_{2}$ for $1 \leq p<3 / 2-\alpha$.

Lemma 3.2. Assume $1 \leq p<3 / 2-\alpha$ and (2.3). Then $\lim _{z \rightarrow 0} I_{2}(z)=0$.

Proof. For $\tau \in\left[\tau_{+}, \tau_{-}\right]$and $q>0$,

$$
\rho_{\alpha}^{q / \alpha}(1-\delta)^{q / \alpha} r^{-q / \alpha} \leq \tau^{-q} \leq \rho_{\alpha}^{q / \alpha}(1+\delta)^{q / \alpha} r^{-q / \alpha},
$$

and so

$$
\begin{aligned}
\left|I_{2}(z)\right| & \leq C \int_{\tau_{+}}^{\tau_{-}} \tau^{-p} \exp \left(-\frac{1}{4} \tau^{-1+2 \alpha}\left|\tau^{-\alpha} z+\gamma_{\alpha}(\tau)\right|^{2}\right) d \tau \\
& \leq C r^{-p / \alpha} \int_{\tau_{+}}^{\tau_{-}} \exp \left(-C^{-1} r^{-(1 / \alpha-2)}\left|\tau^{-\alpha} z+\gamma_{\alpha}(\tau)\right|^{2}\right) d \tau
\end{aligned}
$$

where $C>0$ denotes a generic constant independent of $z$ and $\tau$. Putting

$$
\eta(r):=\max \left\{\sup _{\tau \in\left[\tau_{+}, \tau_{-}\right]}\left|\gamma_{\alpha}(\tau)-v_{\alpha}\right|, r^{1 / 2 \alpha-1}\right\},
$$

we have

$$
\begin{aligned}
\left|\tau^{-\alpha} z+\gamma_{\alpha}(\tau)\right| & \geq\left(\left|\tau^{-\alpha} z+v_{\alpha}\right|-\left|\gamma_{\alpha}(\tau)-v_{\alpha}\right|\right) \chi_{\left\{\left|\tau^{-\alpha} r-\rho_{\alpha}\right| \geq \eta(r)\right\}}(\tau) \\
& \geq\left(\left|\tau^{-\alpha} r-\rho_{\alpha}\right|-\eta(r)\right) \chi_{\left\{\left|\tau^{-\alpha} r-\rho_{\alpha}\right| \geq \eta(r)\right\}}(\tau)
\end{aligned}
$$

for all $\tau \in\left[\tau_{+}, \tau_{-}\right]$. Therefore, by the change of variables $\tau=\left\{r\left(\rho_{\alpha}+\eta(r) \sigma\right)^{-1}\right\}^{1 / \alpha}$,

$$
\begin{aligned}
\left|I_{2}(z)\right| \leq & C r^{-p / \alpha} \int_{\tau_{+}}^{\tau_{-}} \exp \left(-C^{-1} r^{-(1 / \alpha-2)}\left(\left|\tau^{-\alpha} r-\rho_{\alpha}\right|-\eta(r)\right)^{2} \chi_{\left\{\left|\tau^{-\alpha} r-\rho_{\alpha}\right| \geq \eta(r)\right\}}(\tau)\right) d \tau \\
\leq & C r^{-(p-1) / \alpha} \eta(r) \int_{-\rho_{\alpha} \delta / \eta(r)}^{\rho_{\alpha} \delta / \eta(r)}\left(\rho_{\alpha}+\eta(r) \sigma\right)^{-1 / \alpha-1} \\
& \times \exp \left(-C^{-1} r^{-(1 / \alpha-2)} \eta(r)^{2}(|\sigma|-1)^{2} \chi_{\{|\sigma| \geq 1\}}(\sigma)\right) d \sigma \\
\leq & C r^{-(p-1) / \alpha} \eta(r) \int_{-\infty}^{\infty} \exp \left(-C^{-1}(|\sigma|-1)^{2} \chi_{\{|\sigma| \geq 1\}}(\sigma)\right) d \sigma .
\end{aligned}
$$


Conditions $1 \leq p<3 / 2-\alpha$ and $(2.3)$ yield $(2 p-3) /(2 \alpha)+1<0 \quad$ and $\lim _{\tau \downarrow 0}\left(\tau^{-(p-1)}\left|\gamma_{\alpha}(\tau)-v_{\alpha}\right|\right)=0$, and hence

$$
\begin{aligned}
r^{-(p-1) / \alpha} \eta(r) & \leq r^{-(p-1) / \alpha}\left\{\tau_{-}^{p-1} \sup _{\tau \in\left[\tau_{+}, \tau_{-}\right]}\left(\tau^{-(p-1)}\left|\gamma_{\alpha}(\tau)-v_{\alpha}\right|\right)+r^{1 / 2 \alpha-1}\right\} \\
& \leq C\left\{\sup _{\tau \in\left[\tau_{+}, \tau_{-}\right]}\left(\tau^{-(p-1)}\left|\gamma_{\alpha}(\tau)-v_{\alpha}\right|\right)+r^{-(2 p-3) / 2 \alpha-1}\right\} \\
& \rightarrow 0 \quad(r \rightarrow 0) .
\end{aligned}
$$

Thus the lemma follows.

We prepare another estimate of $I_{2}$.

\section{Lemma 3.3. Assume that}

$$
\xi\left(t_{0}\right)-\xi(s)=\left(t_{0}-s\right)^{\alpha} v_{\alpha}+O\left(\left(t_{0}-s\right)^{1 / 2}\right) \quad \text { as } s \uparrow t_{0} .
$$

Then, for any $\delta \in(0,1)$, there exists a constant $C>0$ such that

$$
I_{2}(z) \leq C r^{-(2 p-3) / 2 \alpha-1} \exp \left(-\frac{1}{2} \rho_{\alpha}^{1 / \alpha}(1-\delta)^{1 / \alpha} r^{-(1 / \alpha-2)}(1-\cos \theta)\right)
$$

for all $z \in \mathbf{R}^{N} \backslash\{0\}$.

Proof. One can easily show that the inequality $|a+b|^{2} \geq(1-c)\left(|a|^{2}-\right.$ $|b|^{2} / c$ ) holds for $a, b \in \mathbf{R}^{N}$ and $c>0$. From this and (3.6), we have

$$
\begin{aligned}
\frac{1}{\tau}\left|z+\xi\left(t_{0}\right)-\xi\left(t_{0}-\tau\right)\right|^{2} & \geq \frac{1-\delta}{\tau}\left|z+\tau^{\alpha} v_{\alpha}\right|^{2}-\frac{1-\delta}{\delta \tau}\left|\xi\left(t_{0}\right)-\xi\left(t_{0}-\tau\right)-\tau^{\alpha} v_{\alpha}\right|^{2} \\
& \geq \frac{1-\delta}{\tau}\left\{\left(r-\tau^{\alpha} \rho_{\alpha}\right)^{2}+2 \tau^{\alpha} \rho_{\alpha} r(1-\cos \theta)\right\}-C
\end{aligned}
$$

for some constant $C>0$ independent of $z$ and $\tau$. Moreover, if $\tau \leq \tau_{-}$,

$$
\tau^{-1+\alpha} r \geq \tau_{-}^{-1+\alpha} r=\rho_{\alpha}^{1 / \alpha-1}(1-\delta)^{1 / \alpha-1} r^{-(1 / \alpha-2)} .
$$

Thus

$$
\begin{aligned}
\left|I_{2}(z)\right| \leq & C \exp \left(-\frac{1}{2} \rho_{\alpha}^{1 / \alpha}(1-\delta)^{1 / \alpha} r^{-(1 / \alpha-2)}(1-\cos \theta)\right) \\
& \times \int_{\tau_{+}}^{\tau_{-}} \tau^{-p} \exp \left(-\frac{1-\delta}{4 \tau}\left(r-\tau^{\alpha} \rho_{\alpha}\right)^{2}\right) d \tau
\end{aligned}
$$

Making the substitution $\tau=\left\{\rho_{\alpha}^{-1} r\left(1+r^{\mu} \sigma\right)^{-1}\right\}^{1 / \alpha}(\mu:=1 /(2 \alpha)-1)$ yields 


$$
\begin{aligned}
\int_{\tau_{+}}^{\tau_{-}} \tau^{-p} & \exp \left(-\frac{1-\delta}{4 \tau}\left(r-\tau^{\alpha} \rho_{\alpha}\right)^{2}\right) d \tau \\
= & \alpha^{-1} \rho_{\alpha}^{(p-1) / \alpha} r^{-(2 p-3) / 2 \alpha-1} \int_{-\delta / r^{\mu}}^{\delta / r^{\mu}}\left(1+r^{\mu} \sigma\right)^{(p-1) / \alpha-1} \\
& \times \exp \left(-\frac{1-\delta}{4} \rho_{\alpha}^{1 / \alpha}\left(1+r^{\mu} \sigma\right)^{1 / \alpha-2} \sigma^{2}\right) d \sigma \\
\leq & C r^{-(2 p-3) / 2 \alpha-1} \int_{-\infty}^{\infty} \exp \left(-C^{-1} \sigma^{2}\right) d \sigma
\end{aligned}
$$

Therefore we obtain (3.7).

Let $\kappa \in(\mu, 2 \mu)(\mu:=1 /(2 \alpha)-1)$ and define a set $\tilde{T}$ by

$$
\tilde{T}:=\left\{z \in \mathbf{R}^{N} \backslash\{0\} ; 1-\cos \theta \leq r^{\kappa}\right\} .
$$

When $\xi$ satisfies (2.4), we write $\xi\left(t_{0}\right)-\xi\left(t_{0}-\tau\right)=\tau^{\alpha} v_{\alpha}+\tau^{1 / 2} \hat{v}+\tau^{1 / 2} \zeta(\tau)$ and define $d(z):=\min \left\{\sup _{\tau \in\left[\tau_{+}, \tau_{-}\right]}|\zeta(\tau)|, 1 / 2\right\}$. Then (2.4) yields $d(z) \rightarrow 0$ as $z \rightarrow 0$.

In the next lemma, we examine the behavior of $I_{2}$ on $\tilde{T}$.

LEMMA 3.4. Under the condition (2.4), the following hold.

$$
\begin{aligned}
& \liminf _{z \in \tilde{T}, z \rightarrow 0}\left[r^{(2 p-3) / 2 \alpha+1} e^{(1 / 4)(1+d(z)) J(z)} I_{2}(z)\right] \geq(4 \pi)^{1 / 2} w_{\beta} \alpha^{-1} \rho_{\alpha}^{(2 p-3) / 2 \alpha} e^{-(1 / 4) c_{\alpha}}, \\
& \limsup _{z \in \tilde{T}, z \rightarrow 0}\left[r^{(2 p-3) / 2 \alpha+1} e^{(1 / 4)(1-d(z)) J(z)} I_{2}(z)\right] \leq(4 \pi)^{1 / 2} w_{\beta} \alpha^{-1} \rho_{\alpha}^{(2 p-3) / 2 \alpha} e^{-(1 / 4) c_{\alpha}} .
\end{aligned}
$$

Here $J$ and $c_{\alpha}$ are defined in Theorem 2.2.

Proof. From the inequality $(1-c)\left(|a|^{2}-|b|^{2} / c\right) \leq|a+b|^{2} \leq(1+c)\left(|a|^{2}+\right.$ $\left.|b|^{2} / c\right)\left(a, b \in \mathbf{R}^{N}, c>0\right)$, we have

$$
\begin{aligned}
\frac{1}{\tau} \mid z+ & \xi\left(t_{0}\right)-\left.\xi\left(t_{0}-\tau\right)\right|^{2} \\
= & \frac{1}{\tau}\left|r \omega+\tau^{\alpha} \rho_{\alpha} v_{\alpha}+\tau^{1 / 2} \hat{v}+\tau^{1 / 2} \zeta(\tau)\right|^{2} \\
\geq & \frac{1-d(z)}{\tau}\left|r \omega+\tau^{\alpha} \rho_{\alpha} v_{\alpha}+\tau^{1 / 2} \hat{v}\right|^{2}-\frac{1-d(z)}{d(z)}|\zeta(\tau)|^{2} \\
\geq & (1-d(z))\left\{\left(\tau^{-1 / 2} r-\tau^{-1 / 2+\alpha} \rho_{\alpha}-v_{\alpha} \cdot \hat{v}\right)^{2}\right. \\
& \left.+2 \tau^{-1+\alpha} \rho_{\alpha} r(1-\cos \theta)+2 \tau^{-1 / 2}(n \cdot \hat{v}) r \sin \theta\right\} \\
& +(1-d(z))\left\{|\hat{v}|^{2}-\left(v_{\alpha} \cdot \hat{v}\right)^{2}\right\} \\
& +2(1-d(z)) \tau^{-1 / 2}\left(v_{\alpha} \cdot \hat{v}\right) r(1-\cos \theta)-(1-d(z)) d(z)
\end{aligned}
$$


and, in a similar way,

$$
\begin{aligned}
\frac{1}{\tau} \mid z+ & \xi\left(t_{0}\right)-\left.\xi\left(t_{0}-\tau\right)\right|^{2} \\
\leq & (1+d(z))\left\{\left(\tau^{-1 / 2} r-\tau^{-1 / 2+\alpha} \rho_{\alpha}-v_{\alpha} \cdot \hat{v}\right)^{2}\right. \\
& \left.+2 \tau^{-1+\alpha} \rho_{\alpha} r(1-\cos \theta)+2 \tau^{-1 / 2}(n \cdot \hat{v}) r \sin \theta\right\} \\
& +(1+d(z))\left\{|\hat{v}|^{2}-\left(v_{\alpha} \cdot \hat{v}\right)^{2}\right\} \\
& +2(1+d(z)) \tau^{-1 / 2}\left(v_{\alpha} \cdot \hat{v}\right) r(1-\cos \theta)+(1+d(z)) d(z)
\end{aligned}
$$

Since the inequality

$$
\tau^{-1 / 2} r(1-\cos \theta) \leq \tau_{+}^{-1 / 2} r^{1+\kappa}=\rho_{\alpha}^{1 / 2 \alpha}(1+\delta)^{1 / 2 \alpha} r^{\kappa-\mu}
$$

holds for any $z \in \tilde{T}$ and $\tau \geq \tau_{+}$, we deduce that

(3.8) $\liminf _{z \in \tilde{T}, z \rightarrow 0}\left(I_{2}(z) / I_{3}^{+}(z)\right) \geq w_{\beta} e^{-(1 / 4) c_{\alpha}}, \quad \limsup _{z \in \tilde{T}, z \rightarrow 0}\left(I_{2}(z) / I_{3}^{-}(z)\right) \leq w_{\beta} e^{-(1 / 4) c_{\alpha}}$,

where

$$
\begin{aligned}
I_{3}^{ \pm}(z):=\int_{\tau_{+}}^{\tau_{-}} \tau^{-p} \exp ( & -\frac{1}{4}(1 \pm d(z))\left\{\left(\tau^{-1 / 2} r-\tau^{-1 / 2+\alpha} \rho_{\alpha}-v_{\alpha} \cdot \hat{v}\right)^{2}\right. \\
& \left.\left.+2 \tau^{-1+\alpha} \rho_{\alpha} r(1-\cos \theta)+2 \tau^{-1 / 2}(n \cdot \hat{v}) r \sin \theta\right\}\right) d \tau
\end{aligned}
$$

By the change of variables $\tau=\left\{\rho_{\alpha}^{-1} r\left(1+r^{\mu} \sigma\right)^{-1}\right\}^{1 / \alpha}$, we have

$$
\begin{gathered}
I_{3}^{ \pm}(z)=\alpha^{-1} \rho_{\alpha}^{(p-1) / \alpha} r^{-(2 p-3) / 2 \alpha-1} e^{-(1 / 4)(1 \pm d(z)) J(z)} \\
\times \int_{-\delta / r^{\mu}}^{\delta / r^{\mu}}\left(1+r^{\mu} \sigma\right)^{(p-\alpha-1) / \alpha} e^{-(1 / 4)(1 \pm d(z))\left(J_{1}+J_{2}\right)} d \sigma \\
J_{1}=J_{1}(z, \sigma):=\left\{\rho_{\alpha}^{1 / 2 \alpha}\left(1+r^{\mu} \sigma\right)^{\mu} \sigma-v_{\alpha} \cdot \hat{v}\right\}^{2}, \\
J_{2}=J_{2}(z, \sigma):=2 \rho_{\alpha}^{1 / \alpha}\left\{\left(1+r^{\mu} \sigma\right)^{1 / \alpha-1}-1\right\} r^{-2 \mu}(1-\cos \theta) \\
+2 \rho_{\alpha}^{1 / 2 \alpha}(n \cdot \hat{v})\left\{\left(1+r^{\mu} \sigma\right)^{1 / 2 \alpha}-1\right\} r^{-\mu} \sin \theta .
\end{gathered}
$$

We easily see that $\lim _{z \rightarrow 0} J_{1}(z, \sigma)=\left(\rho_{\alpha}^{1 / 2 \alpha} \sigma-v_{\alpha} \cdot \hat{v}\right)^{2}$ for each $\sigma \in \mathbf{R}$ and

$$
J_{1}(z, \sigma) \geq \frac{1}{2} \rho_{\alpha}^{1 / \alpha}\left(1+r^{\mu} \sigma\right)^{2 \mu} \sigma^{2}-\left(v_{\alpha} \cdot \hat{v}\right)^{2} \geq \frac{1}{2} \rho_{\alpha}^{1 / \alpha}(1-\delta)^{2 \mu} \sigma^{2}-\left(v_{\alpha} \cdot \hat{v}\right)^{2}
$$

provided that $\sigma \geq-\delta / r^{\mu}$. For $z \in \tilde{T}$ and $\sigma \in\left[-\delta / r^{\mu}, \delta / r^{\mu}\right], J_{2}$ is estimated as 


$$
\begin{aligned}
\left|J_{2}(z, \sigma)\right| & \leq C r^{\mu}|\sigma| \cdot r^{-2 \mu}(1-\cos \theta)+C r^{\mu}|\sigma| \cdot r^{-\mu}|\sin \theta| \\
& \leq C\left\{r^{-\mu}(1-\cos \theta)+(1-\cos \theta)^{1 / 2}\right\}|\sigma| \\
& \leq C\left(r^{\kappa-\mu}+r^{\kappa / 2}\right)|\sigma|,
\end{aligned}
$$

where $C>0$ is a constant. This particularly implies that $\lim _{z \in \tilde{T}, z \rightarrow 0} J_{2}(z, \sigma)=0$ for fixed $\sigma \in \mathbf{R}$, and furthermore, there is a constant $C>0$ such that

$$
\left(1+r^{\mu} \sigma\right)^{(p-\alpha-1) / \alpha} e^{-(1 / 4)(1 \pm d(z))\left(J_{1}(z, \sigma)+J_{2}(z, \sigma)\right)} \chi_{\left[-\delta / r^{\mu}, \delta / r^{\mu}\right]}(\sigma) \leq C e^{-C^{-1}\left(\sigma^{2}-\sigma\right)}
$$

for all $z \in \tilde{T}$ with $r \leq 1$ and $\sigma \in \mathbf{R}$. The right-hand side is integrable on $\mathbf{R}$, and so Lebesgue's dominated convergence theorem gives

$$
\begin{aligned}
\lim _{\substack{z \in \tilde{T} \\
z \rightarrow 0}}\left\{r^{(2 p-3) / 2 \alpha+1} e^{(1 / 4)(1 \pm d(z)) J(z)} I_{3}^{ \pm}(z)\right\} \\
\quad=\alpha^{-1} \rho_{\alpha}^{(p-1) / \alpha} \int_{-\infty}^{\infty} \exp \left(-\frac{1}{4}\left(\rho_{\alpha}^{1 / 2 \alpha} \sigma-v_{\alpha} \cdot \hat{v}\right)^{2}\right) d \sigma \\
\quad=(4 \pi)^{1 / 2} \alpha^{-1} \rho_{\alpha}^{(2 p-3) / 2 \alpha} .
\end{aligned}
$$

From this and (3.8), the lemma follows.

We are now in a position to prove Theorem 2.2.

Proof of Theorem 2.2. (i) is a direct consequence of Lemmas 3.1 and 3.2, and therefore it suffices to consider (ii).

In what follows, we suppose $p \geq 3 / 2-\alpha$ and (2.4). First we derive (2.5). For given $\varepsilon>0$, we take $\delta$ so that $(1-\delta)^{1 / \alpha}((2 p-3) /(2 \alpha)+1+\varepsilon) \geq$ $(2 p-3) /(2 \alpha)+1+\varepsilon / 2$. Then, by Lemma 3.3 ,

$$
\begin{aligned}
\left|I_{2}(z)\right| & \leq C r^{-(2 p-3) / 2 \alpha-1} \exp \left(-\frac{1}{2} \rho_{\alpha}^{1 / \alpha}(1-\delta)^{1 / \alpha} r^{-(1 / \alpha-2)}(1-\cos \theta)\right) \\
& \leq C r^{-(2 p-3) / 2 \alpha-1} \exp \left(-\left(\frac{2 p-3}{2 \alpha}+1+\frac{\varepsilon}{2}\right) \log \frac{1}{r}\right) \\
& =C r^{\varepsilon / 2}
\end{aligned}
$$

for all $z \in S_{\varepsilon}$. From this and Lemma 3.1, we conclude (2.5).

Next we show (2.6). Since $\sin \theta \leq\{2(1-\cos \theta)\}^{1 / 2}$, we have $\sup _{z \in T_{M}}|J(z)|$ $<+\infty$. Note that $T_{M} \cap\{|z| \leq \eta\} \subset \tilde{\tilde{T}}$ provided that $\eta>0$ is small. Hence we see from Lemma 3.4 that

$$
I_{2}(z)=(1+o(1))(4 \pi)^{1 / 2} w_{\beta} \alpha^{-1} \rho_{\alpha}^{(2 p-3) / 2 \alpha} e^{-(1 / 4) c_{\alpha}-(1 / 4) J(z)} r^{-(2 p-3) / 2 \alpha-1}
$$

as $z \in T_{M}, z \rightarrow 0$. This and Lemma 3.1 give (2.6). 
(2.7) immediately follows from Fatou's lemma and (2.5), and so we only have to show (2.8). It is easily seen that for any $\varepsilon>0,\left(\mathbf{R}^{N} \backslash \tilde{T}\right) \cap\{|z| \leq \eta\} \subset S_{\varepsilon}$ provided that $\eta$ is small. Therefore (2.5) and (3.5) yield

$$
\begin{aligned}
\limsup _{x \rightarrow \xi\left(t_{0}\right)} & \left(r^{(2 p-3) / 2 \alpha+1} F\left(x, t_{0}\right)\right) \\
= & \limsup _{\substack{x-\xi\left(t_{0}\right) \in \tilde{T} \\
x \rightarrow \xi\left(t_{0}\right)}}\left(r^{(2 p-3) / 2 \alpha+1} F\left(x, t_{0}\right)\right) \\
= & \begin{cases}F\left(\xi\left(t_{0}\right), t_{0}\right)+(4 \pi)^{-N / 2} \limsup _{z \in \tilde{T}, z \rightarrow 0} I_{2}(z) & \text { if } p=\frac{3}{2}-\alpha, \\
(4 \pi)^{-N / 2} \limsup _{z \in \tilde{T}, z \rightarrow 0}\left(r^{(2 p-3) / 2 \alpha+1} I_{2}(z)\right) & \text { if } p>\frac{3}{2}-\alpha .\end{cases}
\end{aligned}
$$

Let us first consider the estimate of the above quantity from below. Set $n_{\alpha}:=-\left\{\hat{v}-\left(v_{\alpha} \cdot \hat{v}\right) v_{\alpha}\right\} /\left|\hat{v}-\left(v_{\alpha} \cdot \hat{v}\right) v_{\alpha}\right|$. This is defined unless $c_{\alpha}=0$ and satisfies $\left|n_{\alpha}\right|=1, n_{\alpha} \cdot v_{\alpha}=0$ and $n_{\alpha} \cdot \hat{v}=-c_{\alpha}^{1 / 2}$. We define

$$
T_{*}:= \begin{cases}\left\{z \in \mathbf{R}^{N} \backslash\{0\} ; n=n_{\alpha}, 2 \rho_{\alpha}^{1 / \alpha}(1-\cos \theta)=c_{\alpha} r^{1 / \alpha-2}\right\} & \text { if } c_{\alpha} \neq 0, \\ \left\{z \in \mathbf{R}^{N} \backslash\{0\} ; \theta=0\right\}=\left\{z \in \mathbf{R}^{N} \backslash\{0\} ; \omega=-v_{\alpha}\right\} & \text { if } c_{\alpha}=0 .\end{cases}
$$

Then it is easy to see that $T_{*} \subset T_{M}$ for large $M$ and $T_{*} \cap\{|z| \leq \eta\} \subset \tilde{T}$ for small $\eta$. Furthermore, since $\lim _{\phi \rightarrow 0}\left\{2(1-\cos \phi) / \sin ^{2} \phi\right\}=1$, we have

$$
\lim _{\substack{z \in T_{*} \\ z \rightarrow 0}} J(z)=c_{\alpha}+2\left(n_{\alpha} \cdot \hat{v}\right) c_{\alpha}^{1 / 2}=-c_{\alpha} .
$$

From this and (3.9), we deduce that

$$
\begin{aligned}
& \limsup _{z \in \tilde{T}, z \rightarrow 0}\left(r^{(2 p-3) / 2 \alpha+1} I_{2}(z)\right) \\
& \quad \geq \lim _{z \in T_{*}, z \rightarrow 0}\left(r^{(2 p-3) / 2 \alpha+1} I_{2}(z)\right)=(4 \pi)^{1 / 2} w_{\beta} \alpha^{-1} \rho_{\alpha}^{(2 p-3) / 2 \alpha} .
\end{aligned}
$$

Next we derive an upper bound. It is elementary to show that $n \cdot \hat{v} \geq$ $-c_{\alpha}^{1 / 2}\left(=n_{\alpha} \cdot \hat{v}\right)$ and that $a \cos \phi+b \sin \phi \leq\left(a^{2}+b^{2}\right)^{1 / 2}$ for $a, b, \phi \in \mathbf{R}$. Hence we have

$$
\begin{aligned}
J(z) & \geq 2 \rho_{\alpha}^{1 / \alpha} r^{-(1 / \alpha-2)}(1-\cos \theta)-2 c_{\alpha}^{1 / 2} \rho_{\alpha}^{1 / 2 \alpha} r^{-(1 / 2 \alpha-1)} \sin \theta \\
& \geq 2 \rho_{\alpha}^{1 / \alpha} r^{-(1 / \alpha-2)}\left\{1-\left(1+c_{\alpha} \rho_{\alpha}^{-1 / \alpha} r^{1 / \alpha-2}\right)^{1 / 2}\right\} \\
& \rightarrow-c_{\alpha} \quad(r \rightarrow 0) .
\end{aligned}
$$

This and Lemma 3.4 give 
(3.12) $\limsup _{z \in \tilde{T}, z \rightarrow 0}\left(r^{(2 p-3) / 2 \alpha+1} I_{2}(z)\right) \leq e^{(1 / 4) c_{\alpha}} \limsup _{z \in \tilde{T}, z \rightarrow 0}\left(r^{(2 p-3) / 2 \alpha+1} e^{(1 / 4)(1-d(z)) J(z)} I_{2}(z)\right)$

$$
\leq(4 \pi)^{1 / 2} w_{\beta} \alpha^{-1} \rho_{\alpha}^{(2 p-3) / 2 \alpha} .
$$

Combining (3.10)-(3.12), we obtain (2.8). Thus the proof is complete.

\section{The profile of $F$ for $\alpha>1 / 2$}

In this section we discuss the effect of $v_{\alpha}$ for $\alpha>1 / 2$. As mentioned in Section 1 , it is known that if $w(t) \equiv 1$ and $\alpha>1 / 2$, the leading term of the expansion of $F\left(x, t_{0}\right)$ as $x \rightarrow \xi\left(t_{0}\right)$ is $\Psi\left(x-\xi\left(t_{0}\right)\right)$. The aim in this section is to obtain the second-order term. More precisely, we prove the following theorem.

THEOREM 4.1. Assume $w(t) \equiv 1$ and $\alpha \in(1 / 2,1]$. Then the following (i), (ii) and (iii) hold as $z=x-\xi\left(t_{0}\right) \rightarrow 0$.

(i) If $N=2$,

$$
\begin{aligned}
F\left(x, t_{0}\right)= & \Psi(z)+(4 \pi)^{-1} \\
& \times\left\{\log \left(4 t_{0}\right)+\Gamma^{\prime}(1)-\int_{0}^{t_{0}} \tau^{-1}\left(1-e^{-(1 / 4) \tau^{2 \alpha-1}\left|\gamma_{\alpha}(\tau)\right|^{2}}\right) d \tau\right\}+o(1) .
\end{aligned}
$$

(ii) If $N=3$ and $\alpha=1$,

$$
\begin{aligned}
F\left(x, t_{0}\right)= & \Psi(z)+(4 \pi)^{-3 / 2} \\
& \times\left[-\frac{2}{\sqrt{t_{0}}}+\Gamma\left(\frac{1}{2}\right) \rho_{\alpha} \cos \theta-\int_{0}^{t_{0}} \tau^{-3 / 2}\left(1-e^{-(1 / 4) \tau\left|\gamma_{1}(\tau)\right|^{2}}\right) d \tau\right]+o(1) .
\end{aligned}
$$

(iii) If $N=3$ and $\alpha \neq 1$, or $N \geq 4$,

$$
F\left(x, t_{0}\right)=\Psi(z)+4^{-(\alpha+1 / 2)} \pi^{-N / 2} \Gamma\left(\frac{N}{2}-\alpha\right) \rho_{\alpha}(\cos \theta) r^{2 \alpha+1-N}+o\left(r^{2 \alpha+1-N}\right) .
$$

Proof. We write

$$
\begin{aligned}
(4 \pi)^{N / 2} F\left(x, t_{0}\right)= & \int_{0}^{t_{0}} \tau^{-N / 2} e^{-r^{2} / 4 \tau} d \tau-\int_{0}^{t_{0}} \tau^{-N / 2} e^{-r^{2} / 4 \tau}\left(1-e^{-(1 / 4) \tau^{2 \alpha-1}\left|\gamma_{\alpha}(\tau)\right|^{2}}\right) d \tau \\
& +\int_{0}^{t_{0}} \tau^{-N / 2} e^{-r^{2} / 4 \tau-(1 / 4) \tau^{2 \alpha-1}\left|\gamma_{\alpha}(\tau)\right|^{2}}\left(e^{-(1 / 2) r \tau^{\alpha-1}\left(\omega \cdot \gamma_{\alpha}(\tau)\right)}-1\right) d \tau \\
= & : I_{1}(z)-I_{2}(z)+I_{3}(z) .
\end{aligned}
$$

We first consider $I_{1}(z)$. The change of variables $\tau=r^{2} / \sigma$ and integration by parts show that as $z \rightarrow 0$, 


$$
\begin{aligned}
I_{1}(z) & =\int_{r^{2} / t_{0}}^{\infty} \sigma^{-1} e^{-(1 / 4) \sigma} d \sigma=\left(\log \frac{t_{0}}{r^{2}}\right) e^{-r^{2} / 4 t_{0}}+\frac{1}{4} \int_{r^{2} / t_{0}}^{\infty} e^{-(1 / 4) \sigma} \log \sigma d \sigma \\
& =2 \log \frac{1}{r}+\log t_{0}+\Gamma^{\prime}(1)+\log 4+O\left(r^{2} \log \frac{1}{r}\right)
\end{aligned}
$$

if $N=2$, and

$$
\begin{aligned}
I_{1}(z) & =\int_{0}^{\infty} \tau^{-N / 2} e^{-r^{2} / 4 \tau} d \tau-\int_{t_{0}}^{\infty} \tau^{-N / 2} e^{-r^{2} / 4 \tau} d \tau \\
& =r^{2-N} \int_{0}^{\infty} \sigma^{N / 2-2} e^{-(1 / 4) \sigma} d \sigma-\frac{2}{(N-2) t_{0}^{N / 2-1}}+O\left(r^{2}\right)
\end{aligned}
$$

if $N \geq 3$.

Next we examine $I_{2}(z)$. Since the integrand of $I_{2}(z)$ is positive and monotone decreasing with respect to $r$, we have,

$$
\lim _{z \rightarrow 0} I_{2}(z)=\int_{0}^{t_{0}} \tau^{-N / 2}\left(1-e^{-(1 / 4) \tau^{2 \alpha-1}\left|\gamma_{\alpha}(\tau)\right|^{2}}\right) d \tau
$$

by the monotone convergence theorem. The right-hand side of the above is finite if $N=2$, or $N=3$ and $\alpha>3 / 4$. If this is not the case, we have $N / 2-2 \alpha-1 \geq-1$, and so

$$
\begin{aligned}
\left|I_{2}(z)\right| & \leq C \int_{0}^{t_{0}} \tau^{-N / 2+2 \alpha-1} e^{-r^{2} / 4 \tau} d \tau=C r^{-N+4 \alpha} \int_{r^{2} / t_{0}}^{\infty} \sigma^{N / 2-2 \alpha-1} e^{-(1 / 4) \sigma} d \sigma \\
& \leq C r^{-N+4 \alpha}\left(1+\log \frac{1}{r}\right),
\end{aligned}
$$

where $C$ denotes a positive constant independent of $z$. In particular, $I_{2}(z)=$ $o\left(r^{-N+2 \alpha+1}\right)$ as $z \rightarrow 0$ if $N \geq 3$ and $\alpha \neq 1$, or $N \geq 4$.

Finally let us consider $I_{3}(z)$. We derive

$$
\lim _{z \rightarrow 0}\left(r^{N-2 \alpha-1} I_{3}(z)-\frac{1}{2} \rho_{\alpha} \cos \theta \int_{0}^{\infty} \sigma^{N / 2-\alpha-1} e^{-(1 / 4) \sigma} d \sigma\right)=0
$$

unless $N=2$ and $\alpha=1$. By the change of variables, we rewrite

$$
\begin{aligned}
I_{3}(z)= & -\frac{1}{2} r \int_{0}^{t_{0}} \tau^{-N / 2+\alpha-1} e^{-r^{2} / 4 \tau-(1 / 4) \tau^{2 \alpha-1}\left|\gamma_{\alpha}(\tau)\right|^{2}}\left(\int_{0}^{1} e^{-(\eta / 2) r \tau^{\alpha-1} \omega \cdot \gamma_{\alpha}(\tau)} d \eta\right) \omega \cdot \gamma_{\alpha}(\tau) d \tau \\
= & -\frac{1}{2} r^{-N+2 \alpha+1} \int_{r^{2} / t_{0}}^{\infty} \sigma^{N / 2-\alpha-1} e^{-(1 / 4) \sigma-(1 / 4) g(\sigma, z)} \\
& \times\left(\int_{0}^{1} e^{-(\eta / 2) f(\sigma, z)} d \eta\right) \omega \cdot \gamma_{\alpha}\left(\frac{r^{2}}{\sigma}\right) d \sigma,
\end{aligned}
$$


where $f(\sigma, z):=r^{2 \alpha-1} \sigma^{1-\alpha} \omega \cdot \gamma_{\alpha}\left(r^{2} / \sigma\right)$ and $g(\sigma, z):=\left|\gamma_{\alpha}\left(r^{2} / \sigma\right)\right|^{2} r^{4 \alpha-2} \sigma^{1-2 \alpha}$. Then,

$$
\begin{aligned}
r^{N-2 \alpha-1} & I_{3}(z)-\frac{1}{2} \rho_{\alpha} \cos \theta \int_{0}^{\infty} \sigma^{N / 2-\alpha-1} e^{-(1 / 4) \sigma} d \sigma \\
= & \frac{1}{2} \int_{0}^{\infty} \sigma^{N / 2-\alpha-1} e^{-(1 / 4) \sigma} \\
& \times\left\{-e^{-(1 / 4) g(\sigma, z)}\left(\int_{0}^{1} e^{-(\eta / 2) f(\sigma, z)} d \eta\right) \omega \cdot \gamma_{\alpha}\left(\frac{r^{2}}{\sigma}\right) \chi_{\left[r^{2} / t_{0}, \infty\right)}(\sigma)+\omega \cdot v_{\alpha}\right\} d \sigma .
\end{aligned}
$$

Since $\lim _{z \rightarrow 0} f(\sigma, z)=\lim _{z \rightarrow 0} g(\sigma, z)=0$ and $\lim _{z \rightarrow 0} \gamma_{\alpha}\left(r^{2} / \sigma\right)=v_{\alpha}$, the integrand of the right-hand side convergences to 0 for any $\sigma \in(0, \infty)$ as $z \rightarrow 0$. Moreover, one easily see that $|f(\sigma, z)| \leq C \sigma^{1-\alpha}$ and $g(\sigma, r) \geq 0$ for $r \in(0,1)$ and $\sigma \in(0, \infty)$, and therefore the integrand is bounded by $C \sigma^{N / 2-\alpha-1} e^{-(1 / 4) \sigma}\left(e^{C \sigma^{1-\alpha}}+1\right)$, which is integrable on $(0, \infty)$ unless $N=2$ and $\alpha=1$. Thus, by applying Lebesgue's dominated convergence theorem, we obtain (4.5).

In the case $N=2$ and $\alpha=1, I_{3}(z)$ is estimated as

$$
\left|I_{3}(z)\right| \leq C r \int_{r^{2} / t_{0}}^{\infty} \sigma^{-1} e^{-(1 / 4) \sigma+C \sigma^{1-\alpha}} d \sigma \leq C r\left(1+\log \frac{1}{r}\right)
$$

with some constant $C>0$. In particular, we see from (4.5) and this computation that $\lim _{z \rightarrow 0} I_{3}(z)=0$ provided that $N=2$.

(i), (ii) and (iii) follow from (4.1)-(4.6). Thus the proof is complete.

\section{REFERENCES}

[1] H. BRÉZIS AND L. VÉRON, Removable singularities for some nonlinear elliptic equations, Arch. Rational Mech. Anal. 75 (1980/81), 1-6.

[ 2 ] B. GidAs AND J. Spruck, Global and local behavior of positive solutions of nonlinear elliptic equations, Comm. Pure Appl. Math. 34 (1981), 525-598.

[3] A. Grigor'YAN, Heat kernel and analysis on manifolds, AMS/IP studies in advanced mathematics 47, American Mathematical Society, Providence, RI, 2009.

[4] K. Hirata, Removable singularities of semilinear parabolic equations, Proc. Amer. Math. Soc. 142 (2014), 157-171.

[5] S. Y. Hsu, Removable singularities of semilinear parabolic equations, Adv. Differential Equations 15 (2010), 137-158.

[6] K. M. HuI, Another proof for the removable singularities of the heat equation, Proc. Amer. Math. Soc. 138 (2010), 2397-2402.

[ 7 ] G. KARCH AND X. Zheng, Time-dependent singularities in the Navier-Stokes system, preprint.

[ 8 ] P.-L. Lions, Isolated singularities in semilinear problems, J. Differential Equations 38 (1980), $441-450$.

[9] S. Sato And E. Yanagida, Solutions with moving singularities for a semilinear parabolic equation, J. Differential Equations 246 (2009), 724-748.

[10] S. SATo AND E. Yanagida, Forward self-similar solution with a moving singularity for a semilinear parabolic equation, Discrete Contin. Dyn. Syst. 26 (2010), 313-331. 
[11] J. Takahashi and E. Yanagida, Removability of time-dependent singularities in the heat equation, preprint.

[12] L. VÉRON, Singular solutions of some nonlinear elliptic equations, Nonlinear Anal. 5 (1981), 225-242.

[13] L. VÉRON, Singularities of solutions of second order quasilinear equations, Pitman research notes in mathematics series 353, Longman, Harlow, 1996.

\author{
Toru Kan \\ Department of Mathematics \\ TOKYO InSTITUTE OF TECHNOLOGY \\ O-OKayama, Meguro-Ku, ToKyo 152-8551 \\ JAPAN \\ E-mail: kan@math.titech.ac.jp \\ Jin Takahashi \\ DEPARTMENT OF MATHEMATICS \\ TOKYO InSTITUTE OF TECHNOLOGY \\ O-OKayama, Meguro-Ku, TOKyo 152-8551 \\ JAPAN \\ E-mail: takahashi.j.ab@m.titech.ac.jp
}

